\title{
Order by disorder and gauge-like degeneracy in quantum pyrochlore antiferromagnet
}

\author{
Christopher L. Henley \\ Laboratory of Atomic and Solid State Physics, Cornell University, Ithaca, New York, 14853-2501
}

\begin{abstract}
The (three-dimensional) pyrochlore lattice antiferromagnet with Heisenberg spins of large spin length $S$ is a highly frustrated model with an macroscopic degeneracy of classical ground states. The zero-point energy of (harmonic order) spin wave fluctuations distinguishes a subset of these states. I derive an approximate but illuminating effective Hamiltonian, acting within the subspace of Ising spin configurations representing the collinear ground states. It consists of products of Ising spins around loops, i.e has the form of a $Z_{2}$ lattice gauge theory. The remaining ground state entropy is still infinite but not extensive, being $O(L)$ for system size $O\left(L^{3}\right)$. All these ground states have unit cells bigger than those considered previously.
\end{abstract}

PACS numbers: PACS numbers:

What is the nature of the ground state of a highly frustrated antiferromagnet with spin length $S$ ? For unfrustrated (and simply frustrated, e.g. triangular) antiferromagnets, a valid recipe is to find the classical ground state (i.e., solution of mean-field theory) and dress it with spin-wave fluctuations. But many frustrated magnets with vector spins have a large classical ground state manifold, having more dimensions than the two or three guaranteed by spin-space rotational symmetry. So, to address frustrated systems with nontrivial ground state degeneracies, one must furthermore identify how quantum fluctuations elect one of the classical states to be the basis of the real ground state (or, alternatively, mix them all, producing a spin-disordered ground state).

Consider the magnon zero-point energy [1] at harmonic or$\operatorname{der}, \mathcal{F}^{\mathrm{h}}\left(\left\{\hat{\mathbf{n}}_{i}\right\}\right) \equiv \sum_{m} \frac{1}{2} \hbar \omega_{m}$, where $\omega_{m}$ runs over all modes of spin waves fluctuating around a particular classical ground state $\mathbf{s}_{i}=S \hat{\mathbf{n}}_{i}$ with unit vectors $\left\{\hat{\mathbf{n}}_{i}\right\}$. [Note that $\mathcal{F}^{\mathrm{h}}$ is defined only on classical ground states: it is just the first term, after the classical one, of an expansion in $1 / S$, and thus dominates in the large- $S$ (semiclassical) limit.] Inequivalent ground states do have different spectra $\left\{\omega_{m}\right\}$, so $\mathcal{F}^{\mathrm{h}}\left(\left\{\hat{\mathbf{n}}_{i}\right\}\right)$ does take different values, and singles out a particular ground state. In simpler cases with (non massive) degeneracies [2] e.g. face-centered cubic (fcc) antiferromagnets $-\mathcal{F}^{\mathrm{h}}\left(\left\{\hat{\mathbf{n}}_{i}\right\}\right)$ is minimized for a unique (modulo rotations) classical configuration $\left\{\hat{\mathbf{n}}_{i}\right\}$ : this long-range ordered state is our answer (for sufficiently large $S$ ). Such breaking of a degeneracy by quantum or thermal fluctuations, or quenched disorder, has been called "order by disorder" [2, 3].

"Highly frustrated" magnets have similar but larger degenerate manifolds, in that the number of independent angle parameters is extensive in the system size. The best-known examples are the two-dimensional kagomé and the threedimensional pyrochlore lattice, built respectively from cornersharing triangles or tetrahedra. My aim here is to ascertain for which classical configuration the quantum fluctuation energy $\mathcal{F}^{\mathrm{h}}$ is smallest; if unique (modulo spin rotation and lattice symmetries), this configuration would give the long-range order of the true ground state.

This paper will address only the $T=0$ ordered state of the large- $S$, pure Heisenberg quantum antiferromagnet with nearest-neighbor couplings on the pyrochlore lattice, which might describe the low- $T$ state that $\mathrm{ZnCr}_{2} \mathrm{O}_{4}(S=3 / 2)$ is approaching before it undergoes a structural transition [4]. Thus our expectation of long-range order does not contradict the evidence for spin-disordered (spin liquid or valence bond crystal) states at $S=1 / 2[5$, 6], or the lack of collinear order as $T \rightarrow 0$ in the classical model [7, 8].

Effective Hamiltonian idea - A major barrier to identifying the selected state is that there is an infinity of states to expand around. This problem is often approached by computing and comparing the $\mathcal{F}^{\mathrm{h}}$ values for special states which have exceptional symmetry [10] or a small magnetic cell [11]. For these special ground states, a numerically exact $\mathcal{F}^{\mathrm{h}}$ can be found by integrating over the magnetic Brillouin zone. Yet in principle we need to know $\mathcal{F}^{\mathrm{h}}$ for each of the continuously infinite ground states, almost all of which are nonperiodic; indeed, the true minimum might not lie in this special subspace [In the present case, it has a larger magnetic cell.]

Instead, my general approach is to derive $\mathcal{F}^{\mathrm{h}}\left(\left\{\hat{\mathbf{n}}_{i}\right\}\right)$ as an effective Hamiltonian [12], for a generic classical ground state, in a crude approximation that has no controlled small parameter, but results in an elegant form (products of spins around loops). This is faithful in the sense that the approximate energies are (mostly) in the same order as the exact ones, and it displays clearly which attributes of a configuration affect its harmonic spin-wave energy.

The collinear states, in the pyrochlore system, are the special subset of states in which $\hat{\mathbf{n}}_{i}=\eta_{i} \hat{\mathbf{n}}$ for all $i$ with $\eta_{i}= \pm 1$. Apart from a global spin axis, they are simply the ground states of the Ising pyrochlore antiferromagnet [13]; this discrete subset still has a massive degeneracy, expressed now as an extensive ground-state entropy $(\sim N \ln (3 / 2))$. I assert (based on many checks, but no proof) that every collinear state is a local minimum of $\mathcal{F}^{\mathrm{h}}$ [14], in accord with the general behavior in exchange-coupled systems [1, 2]; I further conjecture that the optimal $\mathcal{F}^{\mathrm{h}}$ is attained on a collinear state. So we may henceforth limit ourselves to collinear states (an enormous reduction of the set) and seek the discrete effective Hamiltonian $\mathcal{F}^{\mathrm{h}}\left(\left\{\eta_{i}\right\}\right)$.

In the kagomé case the discrete coplanar states [9] play the same role as collinear states do for the pyrochlore; there, it turned out that it every coplanar state has the same spectrum $\left\{\omega_{m}\right\}$, hence $\mathcal{F}^{\mathrm{h}}()$ was independent of the discrete configuration (among coplanar states). A self-consistent anharmonic calculation was required to reduce this degeneracy to a unique state with the final conclusion of long-range order in the $\sqrt{3} \times \sqrt{3}$ state [10, 15]. Note the independence of the spec- 
trum depended on the fact that $\hat{\mathbf{n}}_{i} \cdot \hat{\mathbf{n}}_{j}=-1 / 2$ for neighbor spins in every coplanar kagomé state.

On the other hand, in collinear pyrochlore states, $\hat{\mathbf{n}}_{i} \cdot \hat{\mathbf{n}}_{j}=$ \pm 1 , hence the classical degeneracy is split by $\mathcal{F}^{\mathrm{h}}$. The goal of this paper is to discover how it is split: what is the (approximate) analytic form of $\mathcal{F}^{\mathrm{h}}$, what is its energy scale, which spin pattern gives the minimum $\mathcal{F}^{\mathrm{h}}$, and how large is the remaining degeneracy. It will be shown that there is an infinite (discrete) number of minimum-energy states, but the groundstate entropy is not extensive in the system size.

Hamiltonian and equation of motion - The pyrochlore lattice is the medial lattice of a diamond lattice, i.e. the pyrochlore sites are the midpoints of the diamond bonds. Every tetrahedron of sites in the pyrochlore lattice is centered by a diamond site. With $N$ unit cells, there are $N_{s}=4 N$ spins. Our Hamiltonian contains only isotropic, antiferromagnet exchange coupling between nearest neighbor spins $\mathbf{s}_{i}$ of length $S$ on the pyrochlore lattice: $\mathcal{H}=J \sum_{\langle i j\rangle} \mathbf{s}_{i} \cdot \mathbf{s}_{j}$, where " $\langle i j\rangle$ " counts each neighbor pair just once.

Now, define the tetrahedron spin $\mathbf{L}_{\alpha} \equiv \sum_{i \in \alpha} \mathbf{s}_{i}$ where $\alpha$ (and other Greek indices) runs over the tetrahedron centers, and " $i \in \alpha$ " means $i$ is one of the four sites in tetrahedron $\alpha$. Then the Hamiltonian can be rewritten $\mathcal{H}=\frac{1}{2} J \sum_{\alpha} \mathbf{L}_{\alpha}^{2}$ and the classical ground states are the (very many) states satisfying $\mathbf{L}_{\alpha}=0$.

The $\left\{\omega_{m}\right\}$ which enter $\mathcal{F}^{\mathrm{h}}$ are the same as the eigenfrequencies of the (linearized) classical dynamics. The classical equations of motion are

$$
\hbar \dot{\mathbf{s}}_{i}=S^{-1} \mathbf{s}_{i} \times \mathbf{h}_{i}=J S^{-1} \mathbf{s}_{i} \times \sum_{\alpha: i \in \alpha} \mathbf{L}_{\alpha}
$$

where $\mathbf{h}_{i} \equiv \delta \mathcal{H} / \delta \mathbf{s}_{i}=J \sum_{\langle i j\rangle} \mathbf{s}_{j}=J \sum_{\alpha: i \in \alpha}\left(\mathbf{L}_{\alpha}-\mathbf{s}_{i}\right)$. In explaining the slow dynamics of the classical system, Ref. [8] had noted that 11 implies

$$
\hbar \dot{\mathbf{L}}_{\alpha}=-S^{-1} J \sum_{\beta} \mathbf{s}_{i(\alpha \beta)} \times \mathbf{L}_{\beta}
$$

where the sum is over the four tetrahedra $\beta$ that are nearest to $\alpha$, and $i(\alpha \beta)$ denotes the (unique) spin shared by $\alpha$ and $\beta$. Without loss of generality, take $\mathbf{s}_{i}{ }^{(0)} \equiv \eta_{i} S \hat{\mathbf{z}}$ in our (assumed) collinear state, and parametrize the deviations as $\delta \mathbf{L}_{\alpha}(t)=$ $\left(\delta L_{\alpha x}(t), \delta L_{\alpha y}(t), 0\right)$ : the linearized spin dynamics reduces to

$$
\delta \dot{\mathbf{L}}_{\alpha}=-S J \sum_{\beta} \mu_{\alpha \beta} \hat{\mathbf{z}} \times \delta \mathbf{L}_{\beta}
$$

in which a $2 N \times 2 N$ matrix $\boldsymbol{\mu}$ is defined with elements $\mu_{\alpha \beta} \equiv \eta_{i(\alpha \beta)}$. So, via the trick of using tetrahedron spins, the dynamical matrix is the classical spin configuration; Now, if we can only massage the formulation so it appears as a perturbation, the expansion will generate an effective Hamiltonian.

Trace expansion and loop effective Hamiltonian - Iterating (3) gives the eigenvalue equation

$$
(\hbar \omega)^{2} \delta \mathbf{L}_{\alpha}=-\hbar^{2} \delta \ddot{\mathbf{L}}_{\alpha}=(S J)^{2} \sum_{\gamma}\left(\boldsymbol{\mu}^{2}\right)_{\alpha \gamma} \delta \mathbf{L}_{\gamma}
$$

TABLE I: Exact versus approximate zero-point energy, as a multiple of $J S N_{s}$. The magnetic unit cell has $N_{\text {mag }}$ spins. Last column is from Eq. (7) with $A=4$, from the expansion of (5) to $O\left(\mathbf{M}^{4}\right)$.

\begin{tabular}{|llll|ll|}
\hline State & $N_{\text {mag }}$ & $\left\langle\tau_{r}\right\rangle$ & $\left\langle\tau_{r} \tau_{s}\right\rangle$ & $\mathcal{F}_{\text {exact }}^{\mathrm{h}}$ & $A=4$ \\
\hline a,b & 4,8 & +1 & +1 & 0.4498 & 0.439 \\
c & 32 & -1 & +1 & 0.4245 & 0.414 \\
d & 64 & 0 & 0 & 0.4460 & 0.430 \\
\hline
\end{tabular}

A square root and trace now express the desired effective Hamiltonian [16, 17]:

$$
\mathcal{F}^{\mathrm{h}}\left(\left\{\eta_{i}\right\}\right) \equiv \frac{1}{2} \sum_{m} \hbar \omega_{m} \equiv \frac{1}{2} J S \operatorname{Tr}\left(\left[\boldsymbol{\mu}^{2}\right]^{1 / 2}\right) .
$$

The matrix has constant diagonal elements, $\left(\boldsymbol{\mu}^{2}\right)_{\alpha \alpha}=4$, so let us break it up as $\boldsymbol{\mu}^{2}=4 \mathbf{I}+\mathbf{M}$, where $\mathbf{I}$ is the identity matrix; $\mathbf{M}$ has elements of form $\eta_{i} \eta_{j}$ and only connects (nearest) even sites of the diamond lattice. Next, insert into (5) a formal Taylor expansion of $(4 \mathbf{I}+\mathbf{M})^{1 / 2}$ Every term $\operatorname{Tr}\left(\mathbf{M}^{n}\right)$ is a product $\prod_{k=1}^{2 n} \eta_{i_{k}}$ over a closed walk of $2 n$ steps on the diamond lattice. A step retraced twice contributes a trivial factor $\eta_{i}^{2}=1$. Thus

$$
\mathcal{F}^{\mathrm{h}}=\mathcal{F}_{0}^{\mathrm{h}}-\sum_{\Gamma} K_{\Gamma} \prod_{i \in \Gamma} \eta_{i}
$$

where $\Gamma$ runs over loops (without acute angles) in the pyrochlore lattice. Eq. (6) has exactly the form of an Ising lattice gauge model [18] on a diamond lattice. The shortest loops $\Gamma$ are 6-step and 8-step loops, which appearing first in the $\operatorname{Tr}\left(\mathbf{M}^{3}\right)$ and $\operatorname{Tr}\left(\mathbf{M}^{4}\right)$ terms (respectively) of the expansion. Such a hexagon and puckered octagon are outlined in Fig. 1. call their coefficients $K_{6}$ and $K_{8}$.

We can rewrite (6) in a more convenient (for some purposes) form. Let $\tau_{r} \equiv \prod_{i \in \text { hexagon } r} \eta_{i}= \pm 1$, the product around one of the hexagons. This is a convenient set of variables, since distinct but "gauge" equivalent Ising states map to the same configuration of $\tau_{r}$ 's, and any product around a longer loop can be written in terms of $\tau_{r}$ 's. We place the value $\tau_{r}$ at the center of each its hexagon; these sites form a new pyrochlore lattice complementary to the old one (and having the same lattice constant). In this notation, (6) becomes [19]

$$
\mathcal{F}^{\mathrm{h}}=-K_{6} \sum_{r} \tau_{r}-\frac{1}{2} K_{8} \sum_{\langle r, s\rangle} \tau_{r} \tau_{s}+\ldots
$$

This has the appearance of an Ising Hamiltonian with an external field $K_{6}<0$, and a coupling $K_{8}>0$; both terms are satisfied by $\tau_{r} \equiv-1$, i.e. every hexagon has a negative loop product. Configurations of $\left\{\eta_{i}\right\}$ that realize this and satisfy the classical ground state condition, $\sum_{i \in \alpha} \eta_{i}=0-$ which do indeed exist - are the ground states to harmonic order [i.e. to $O(J S)$ ]. Fig. 11 c) shows the simplest harmonic ground state to construct, with 32 spins in the magnetic unit cell; the smallest cell has 16 spins. [All previously studied cases were $Q=0$ states with a 4-spin magnetic cell, for which the collinear state always has $\prod_{i \in \Gamma} \eta_{i}=+1$, the worst case.] 
In Table the numerically exact zero point energy is compared to (6), taken to order $\mathbf{M}^{4}$. The configurations $\left\{\eta_{i}\right\}$ were constructed to have different average values (as listed) of the terms in (7). Configurations $(a, b, c)$ are shown in Fig. 1] A fit based on just the three exact energies in Table \would give $K_{6}^{\mathrm{fit}}=-1.3 \times 10^{-2}$ and $K_{8}^{\mathrm{fit}}=+1.8 \times 10^{-2}$. The $O\left(\mathbf{M}^{3}\right)$ analytic result is $K_{6}^{\text {th }}=-3 / 2^{8}=-1.2 \times 10^{-2}$, and the $O\left(\mathbf{M}^{4}\right)$ term gives $K_{8}^{\text {th }}=15 / 2^{11}=+0.7 \times 10^{-2}$.

It will be seen that a term in (6) for a $2 n$-step loop actually gets contributions from infinitely many terms in the expansion of (5), as terms in $\mathbf{M}^{m}$ with $m>n$ can represent the loop $\Gamma$ plus retraced steps. In a sense, the higher powers of $\boldsymbol{\mu}$ contain additional contributions of $\mathbf{I}$; one might speculate that a different breakup, $\boldsymbol{\mu}^{2}=4 A \mathbf{I}+\mathbf{M}^{\prime}$, is more accurate. As the spectrum of $\boldsymbol{\mu}^{2}$ fills the interval $[0,16]$, one choice is to expand around its center, i.e. $4 A=8$, to optimize the convergence properties; the original choice $4 A=4$ is also natural since $\mathbf{M}^{\prime}$ has no diagonal terms. As an ad-hoc compromise, I suggest the geometric mean $A=\sqrt{2}$; exactly that value turned out to be optimal in a subsequent, systematic graphical resummation, using a Bethe lattice approximation [16]. [Those numerical energies agreed to $\sim 1 \%$ with numerically exact integration of the zero-point energy for a large sample of classical ground states [16].]

Gauge-like symmetry and ground-state degeneracy Eq. (5) has an exact "gauge" invariance. Let $\eta_{i(\alpha, \beta)}^{\prime}=$ $u_{\alpha} u_{\beta} \eta_{i(\alpha, \beta)}$ where $u_{\alpha}= \pm 1$ arbitrarily; in matrix notation, $\boldsymbol{\mu}^{\prime}=\mathbf{U} \boldsymbol{\mu} \mathbf{U}^{-1}$, where $\mathbf{U}=\operatorname{diag}\left(\left\{u_{\alpha}\right\}\right)$. Thus $\boldsymbol{\mu}^{\prime}$ is similar to $\boldsymbol{\mu}$, the two configurations have the same harmonic eigenvalue spectrum and exactly the same value for $\mathcal{F}^{\mathrm{h}}\left(\left\{\eta_{i}\right\}\right)$ : in other words, this is a generic way to make $\left\{\eta_{i}^{\prime}\right\}$ degenerate with $\left\{\eta_{i}\right\}$. I place the term "gauge" in quotes, since genuinely gauge-equivalent configurations are the same state which has been labeled redundantly, whereas here they are distinct quantum states. Note too that we must uphold an independent (non-"gauge"-invariant) condition that $\sum_{i \in \alpha} \eta_{i}=0$ in every tetrahedron, so this spin configuration is still a classical ground state.

We can construct a relatively simple family of ground states of $\mathcal{F}^{\mathrm{h}}$. A slice (as marked in Fig. 11) has sites with four different $z$ values, constant along the diagonal lines. Let the $\eta_{i}$ along each diagonal line $\ell$ be alternating in sign; to fix an overall sign for each, choose one $\langle 110\rangle$ and one $\langle 1 \overline{1} 0\rangle$ plane normal to the lines, and let $\nu_{\ell}$ be the spin in that layer. Then, the extreme tip sites (as seen in projection) of a hexagon lie on two lines $\ell, \ell^{\prime}$ at the same $z$; the remaining sites are two pairs (lying on lines in the other diagonal direction). Hence, the loop product for that hexagon is $\tau_{s} \equiv \prod_{i \in s} \eta_{i}=\nu_{\ell} \nu_{\ell^{\prime}}$. If we choose $\left\{\nu_{\ell}\right\}$ to alternate between adjacent lines in the same $z$-layer, we ensure that $\tau_{r} \equiv-1$ for all hexagons as e.g. in Fig. 1 c). [Configuration (d) in Table \is a layered state, with signs $\nu_{\ell}$ alternating in each layer of spins along [110] lines but all signs $\nu_{\ell}=+1$ for the lines of spins in the [110] direction and hence not a harmonic ground state.] There is still an arbitrary sign choice in each $z$-layer, so this construction gives $2^{Z}$ distinct ground states, where $Z$ is the number of distinct layers (separated by $\Delta z=a / 4$ in terms of the cubic lattice constant $a$ ). This gives a lower bound of $O(L)$ for the (a)

(b)
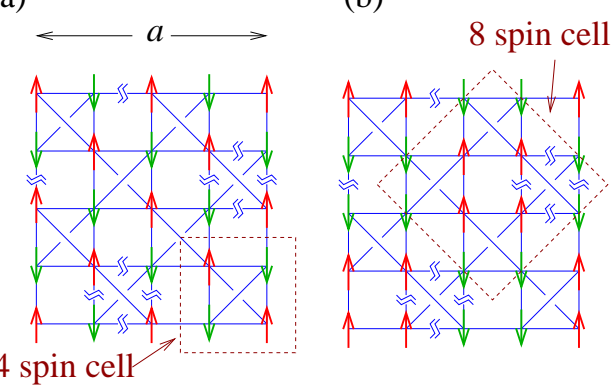

(c)

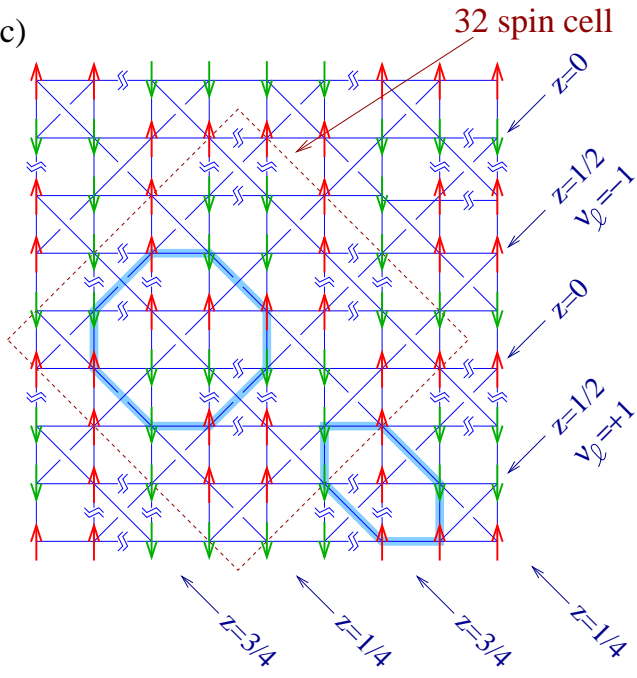

FIG. 1: [COLOR ONLINE]. Spin configurations on the pyrochlore lattice. Sites shown lie in a slice of thickness one unit cell, normal to the page, and all these configurations repeat in that direction. The hexagon and puckered octagon loops are superposed (dashed). The diagonal lines lie in the plane of the page, forming four families at different heights $z$ (marked). Lines running in the $x$ or $y$ directions are tilted $45^{\circ}$ from the vertical, and are shown broken where they connect to an adjacent slice. The lattice unit cell (outlined in (a)) contains 4 spins and has lattice constant $a$. Configurations (a) and (b) are "gauge" equivalent and have the highest possible harmonic zero-point energy $\mathcal{F}^{\mathrm{h}}$. Configuration (c), with its 32 -spin magnetic cell outlined, is the simplest one of many that have the lowest $\mathcal{F}^{\mathrm{h}}$. [Energies for these configurations are in Table凹]

harmonic-ground-state entropy (in a cube of volume $L^{3}$ ), so the entropy per spin is zero in the thermodynamic limit.

We do not yet understand the full set of ground states; note that "gauge" equivalent states exist that are not given by the layer construction [16], and the total entropy was proven to have an upper bound [16] of $O(Z \ln Z)$, only logarithmically larger than the lower bound.

Discussion - In summary, I have computed the $O(J S)$ spin energy term which (partly) breaks a classical degeneracy in the large- $S$ pyrochlore antiferromagnet with purely isotropic interactions. Various complex orderings are found in real pyrochlore antiferromagnets, most often explained by elastic distortions or dipolar interactions [20] or - less interestingly - by nonnegligible second-neighbor exchange. It was nevertheless valuable to isolate the role of quantum fluctuations here, since materials may be found in which those per- 
turbations are very small [21], or frustrated Heisenberg models may be cleanly realized by cold gases in optical traps [22].

Two tricks - the Moessner-Chalker equation of motion, Eq. (2) and writing the zero-point energy as the trace of a matrix, Eq. (5) - enabled an (uncontrolled) expansion of the $O(J S)$ term of the energy to give the effective Hamiltonian $\mathcal{F}^{\mathrm{h}}$. The result (6) is a sum of products of Ising spins around loops. The problem has an exact "gauge" degeneracy implying a ground state entropy, to harmonic order, of (at least) $O(L)$. To fully resolve the degeneracy, as in the kagomé case [10, 15], anharmonic spin-wave calculations are in progress [23].

It was plausible that the quantum system shows greater order than the classical one; what is surprising is that the pyrochlore is less ordered than the kagomé Heisenberg antiferromagnet in the classical $T \rightarrow 0$ case [8], but more ordered in the quantum large- $S$ case, since harmonic fluctuations remove an extensive degeneracy in the pyrochlore but not the kagomé case.

The loop effective Hamiltonian has already been applied to other systems [24]. A similar approach made it possible to determine the ground state within the large- $N$, large- $S$ approximation of the pyrochlore [25]. A more complete analytic derivation [16] of (6) matches numerical fits to $\sim 1 \%$; it also highlights the modes (fewer than in the kagomé case) which have divergent fluctuations in the harmonic approximation.

An effective Hamiltonian such as (6) has value beyond the possibility (as here) that it leads us to an unexpected ground state. It can also be plugged in to define a Boltzmann distribution, such as $\exp \left(-\beta \mathcal{F}^{\mathrm{h}}\right)$, which at low but nonzero temperatures is more valid than the classical spin ensemble [12]. It also a gives basis on which to build more complete or more realistic models, by the addition of anisotropies, quantum tunneling [26], or dilution [12, 27]. Notice how the collinear selection has provided a different route than "spin ice" to realize an effective Ising model in a pyrochlore system; furthermore, tunneling between collinear states [26] might realize ring exchanges [28] (and the consequent $U(1)$ spin liquid).

Acknowledgments - I thank M. Kvale, E. P. Chan, R. Moessner, B. Canals, H. Tsunetsugu, O. Tchernyshyov, P. Sharma, and especially U. Hizi for discussions. This work was supported by NSF Grant No. DMR-0240953.
[1] E. F. Shender, Sov. Phys. JETP, 56, 178 (1982).

[2] C. L. Henley, J. Appl. Phys. 61, 3962 (1987); C. L. Henley, Phys. Rev. Lett., 62, 2056 (1989).

[3] J. Villain et al, J. Phys. (Paris) 41, 1263 (1980).

[4] S.-H. Lee et al Nature 418, 856 (2002).

[5] A.B. Harris, A. J. Berlinsky, and C. Bruder, J. Appl. Phys. 69, 5200 (1991).

[6] B. Canals and C. Lacroix, Phys. Rev. Lett. 80, 2933 (1998); Phys. Rev. B 61, 1149 (2000).

[7] J. N. Reimers, Phys. Rev. B 45, 7287 (1992).

[8] R. Moessner and J. T. Chalker, Phys. Rev. Lett. 80, 2929 (1998); Phys. Rev. B 58, 12049 (1998).

[9] I. Ritchey, P. Coleman and P. Chandra, Phys. Rev. B, 47, 15342 (1993).

[10] A. V. Chubukov, Phys. Rev. Lett., 69, 832 (1992). See also A. Chubukov, J. Appl. Phys., 73, 5639 (1993).

[11] M. Kvale, unpublished (1993); R. R. Sobral and C. LaCroix, Sol. State. Comm. 103, 407 (1997); H. Tsunetsugu, J. Phys. Soc. Jpn. 70, 640 (2001).

[12] C. L. Henley, Can. J. Phys. 79, 1307 (2001).

[13] P. W. Anderson, Phys. Rev. 102, 1008 (1956),

[14] I note that $\sum \omega_{m}^{2} \propto \operatorname{Tr}\left(\boldsymbol{\mu}^{2}\right)$, where $\boldsymbol{\mu}$ is a matrix defined later in the paper. This sum is quickly shown to be independent of which ground state $\left\{\hat{\mathbf{n}}_{i}\right\}$ is (collinear or not). But $\sum \omega^{4}$ attains (the same) maximum for all collinear states, and is reduced by any deviations of $\left\{\hat{\mathbf{n}}_{i}\right\}$, consonant with the arguments in Ref. 2 that collinearity generically maximizes the spectrum's dispersion. That makes it plausible that $\mathcal{F}^{\mathrm{h}} \propto \sum|\omega|$ has a minimum.

[15] E. P. Chan, Ph. D. thesis (Cornell Univ., 1994); J. Mag. Mag. Mater. 140-144, 1693 (1995).

[16] U. Hizi and C. L. Henley, preprint.
[17] The $2 N$ deviations $\left\{\delta \mathbf{L}_{\alpha}\right\}$ are linear combinations of the $4 N$ deviations $\left\{\delta \mathbf{s}_{i}\right\}$, so every eigenfrequency $\omega_{m}$ of the former is a spinwave frequency of the latter. Furthermore, it can be shown (Ref. 16) that every mode lost in the projection is a zerofrequency mode that does not affect $\mathcal{F}^{\mathrm{h}}$.

[18] J. B. Kogut, Rev. Mod. Phys. 51, 659 (1979).

[19] There are $N_{s}$ hexagons and $N_{s} / 2$ octagon loops; each of the latter (see Ref. [16]) corresponds to two $\tau_{r} \tau_{s}$ terms.

[20] J. N. Reimers et al, Phys. Rev. B 43, 3387 (1991); S.-H. Lee et al, Phys. Rev. Lett. 84, 3718 (2000); J. D. M. Champion et al, Phys. Rev. B 64, 140407 (2001); J. D. M. Champion et al, Phys. Rev. B 68, 020401 (2003); O. Tchernyshyov, R. Moessner, and S. L. Sondhi, Phys. Rev. Lett. 88, 067203 (2002).

[21] K. Awaga et al, Phys. Rev. B 49, 3975 (1994); K. Hida, J. Phys. Soc. Jpn. 69, 4003 (2000).

[22] L. Santos, M. A. Baranov, J. I. Cirac, H.-U. Everts, H. Fehrmann, and M. Lewenstein, Phys. Rev. Lett. 93, 030601 (2004).

[23] U. Hizi and C. L. Henley, unpublished.

[24] O. Tchernyshyov, et al Phys. Rev. B 68, 144422 (2003); O. Tchernyshyov, H. Yao, and R. Moessner, Phys. Rev. B 69, 212402 (2004); O. Tchernyshyov, J. Phys.: Condens. Matter 16, S709 (2004).

[25] U. Hizi, P. Sharma, and C. L. Henley, cond-mat/0506574

[26] J. von Delft and C. L. Henley, Phys. Rev. B 48, 965 (1993).

[27] E. F. Shender, V. B. Cherepanov, P. C. W. Holdsworth and A. J. Berlinsky, Phys. Rev. Lett., 70, 3812 (1993).

[28] M. Hermele, M. P. A. Fisher, and L. Balents Phys. Rev. B 69, 064404 (2004). 